\title{
Modelo numérico para el análisis y el diseño de redes de tubería para flujo bifásico
}

\section{Numerical model for the analysis and design of piping networks for two-phase flow}

\author{
Gustavo Valle-Tamayo ${ }^{1}$, Leonardo Valbuena-Luna ${ }^{2}$, Carlos Rojas-Beltrán ${ }^{3}$, Manuel Cabarcas-Simancas ${ }^{4}$ \\ ${ }^{1}$ Petroleum Consulting Company S.A.S., Grupo de Modelamiento de Procesos de Hidrocarburos. \\ Email: gustavo.valle@pccsas.com \\ ${ }^{2}$ Petroleum Consulting Company S.A.S. Email: Leonardo.valbuena@pccsas.com \\ ${ }^{3}$ Petroleum Consulting Company S.A.S. Email: carlos.rojas@ pccsas.com \\ ${ }^{4}$ Grupo de Modelamiento de Procesos de Hidrocarburos, Universidad Industrial de Santander. Email: cabarcas@uis.edu.co
}

Recibido: mayo 24, 2017. Aceptado: marzo 10, 2018. Versión final: abril 14, 2018.

\begin{abstract}
Resumen
Se presenta aquí un nuevo algoritmo para analizar flujo bifásico en redes de recolección. Este algoritmo se fundamenta en la resolución en marcha de las variables hidráulicas, siendo una modificación al propuesto inicialmente por Tian y Adewum, quienes consideraron la fase liquida como una unidad de solo aceite, a diferencia del presente estudio, que, en adición, contempla agua. Para su implementación, no hay necesidad de generar arreglos matriciales complejos, ya que adopta el esquema de las reglas de Kirchhoff. Asimismo, se modificaron algunas correlaciones de flujo, para que se adaptaran al algoritmo propuesto. El algoritmo se plantea utilizando la correlación de Beggs y Brill, en un caso ilustrativo.
\end{abstract}

Palabras clave: algoritmo solución; flujo bifásico; redes de tubería.

\begin{abstract}
A new algorithm for analyzing biphasic flow in collection networks is presented. This algorithm is based on the current resolution of the hydraulic variables, being a modification to the principle proposed by Tian and Adewum, who considered the liquid phase as a unit of water alone, unlike the present study which also considers oil. For its implementation, there is no need to generate complex matrix arrays, since it adopts Kirchhoff's philosophy of rules. Also, some flow correlations have been modified to fit the proposed algorithm. The algorithm is tested with the Beggs and Brill correlation in an illustrative case.
\end{abstract}

Keywords: biphasic flow; pipe networks; solution algorithm.

\section{Introducción}

Extensivas investigaciones para flujo bifásico en tuberías han sido tratadas por más de 60 años. Valle et al. [1] realizan un detallado estado del arte, respecto a los modelos matemáticos enfocados a la caracterización

hidráulica del flujo multifásico. Sin embargo, en la literatura, la aplicación de esos métodos ha sido limitada a esquemas constituidos por solo una línea de flujo, exceptuando las herramientas comerciales que permiten la implementación en redes de tuberías. 
En la búsqueda bibliográfica efectuada para esta investigación (p. ej. Scientdirect, Onepetro, Google Academic), son escasos los métodos satisfactorios que se han reportado para analizar y diseñar redes de flujo bifásico, gas-líquido. No obstante, los autores Mucharam y Adewumi [2] y Tian y Adewumi [3] lograron modelar sistemas complejos de tuberías con flujo bifásico, pero su aplicabilidad se ve restringida, ya sea por el tipo de configuración del sistema, ya sea por el tipo de fluido tratado (i.e. gas y aceite).

Por su parte, Shamir y Howard [4] publicaron un método para el análisis de flujo monofásico en redes, haciendo uso del método iterativo de Newton-Raphson, para resolver el conjunto de ecuaciones resultante del balance de masa. La aplicación de esta técnica a sistemas de flujo monofásico es reconocida en la literatura; no obstante, no ha sido replanteada para sistemas bifásicos, probablemente debido a la complejidad implicada en la obtención de las derivadas necesarias para su correcta puesta en marcha.

El presente trabajo tiene como objetivo plantear un modelo numérico para flujo bifásico, basado en el propuesto por Tian y Adewumi [3], el cual ha sido modificado con el fin de superar las dificultades adscritas a este. En primera medida esto se efectuó mediante la consideración de una mezcla de agua y aceite como fase líquida complementaria a la fase gaseosa, y a través de la implementación de diversos tipos de correlaciones de flujo multifásico (p. ej. [5, 6, 7]). Finalmente, es necesario resaltar que este modelo no requiere un arreglo matricial, ya que está basado en un algoritmo de solución simultánea que facilita su aplicación. Asimismo, se presentan los diversos modelos numéricos para el modelado de redes de flujo monofásico, descritos en la literatura, que permitirán sustentar el fundamento matemático del algoritmo propuesto.

\section{Modelos de redes de tuberías}

El objetivo principal del modelado enfocado a la construcción de redes de tuberías es simular el comportamiento hidráulico de los fluidos. Un esquema típico usado en muchos problemas de mecánica de fluidos es el modelo en estado estacionario, el cual consiste en establecer condiciones estables en la red, en un instante/punto del tiempo [8]. Cuando se conocen puntos de presión y flujo del modelo, se pueden implementar ecuaciones de continuidad (balance de masa) y balance energético para estimar el caudal y la presión en cualquier punto del sistema. Varios métodos numéricos de resolución de problemas se han desarrollado para resolver el modelo en estado estacionario.

A continuación, se presentan algunos de los métodos de solución numérica para sistemas conformados por esquemas monofásicos, los cuales serán insumo para el modelo propuesto.

\section{Modelos de solución numérica}

La complejidad de modelar redes de recolección se fundamenta en el requerimiento de caudal y presión en todas las tuberías y nodos del sistema. La conceptualización del problema demanda características físicas de las tuberías, y la especificación de las variables presión y caudal dentro del sistema es un desafío, al intentar representar el comportamiento hidráulico del fluido cuando diferentes corrientes fluyen a un mismo nodo, para lo cual, en esta investigación, se suscitan estudios enmarcados en la literatura, cuyo objeto sea la resolución del flujo de fluidos en sistemas tipo bucles. Estos son un punto de partida para comprender el análisis matemático y numérico, relacionado con la problemática evidenciada.

Con el fin de resolver el problema de la red de fluidos en bucles, es muy común el empleo de las dos leyes de Kirchhoff, desarrolladas originalmente para resolver circuitos eléctricos, las cuales son equivalentes a las ecuaciones de continuidad y conservación de la energía, respectivamente [9], y establecen lo siguiente: 1) la suma algebraica de las corrientes en un nodo pertenecientes a una red de conductores es cero; 2) la suma dirigida de las diferencias de potencial eléctrico (i.e. voltios) alrededor de cualquier circuito cerrado es cero.

Aplicando las anteriores leyes al problema de red de tuberías en bucle, la corriente eléctrica en la primera ley es equivalente al flujo de masa, y el potencial eléctrico en la segunda ley corresponde a la caída de presión. Por lo tanto, una de las leyes se satisface en el modelado del problema y la otra, lo que resuelve un sistema no lineal de ecuaciones [10]. Basándose en la linealización de este sistema de ecuaciones, Cross [10] propuso un método de resolución, que es esencialmente un método de relajación adecuado para solventar el problema manualmente [11], y así obtener los métodos de modelado, denominados: Método de Flujo Equilibrado y el Método de Presión Balanceada, que satisfacen la primera y segunda ley de Kirchhoff, respectivamente [10].

En busca de mejorar la resolución del problema, se han desarrollados métodos basados en 1) la partición de la matriz representativa del problema $[12,13]$; 2) la linealización [14], y 3) Newton-Raphson para resolver el problema de la red de fluidos en bucles para el método de Hardy-Cross [15, 16]. Para esta última, las características de las ecuaciones y las suposiciones iniciales de las variables dependientes definen la propiedad de convergencia del problema. 
Atendiendo a lo anterior, se procederá a describir los métodos de resolución mencionados.

\subsection{Hardy-Cross}

La idea básica del método de Hardy-Cross es que la conservación de la masa en cada nodo puede establecerse inicialmente [10]. Esto significa que primero se debe asumir una estimación inicial de los flujos en cada elemento de la tubería antes de iniciar el cálculo de la caída de presión.

Para cualquier tubería en el que $Q_{0}$ es asumido como el flujo inicial, la ecuación 1 (i.e. ecuación característica de la tubería) puede ser estimada usando una expansión de la serie Taylor, como sigue en la ecuación 2:

$$
\begin{gathered}
h_{f}=K Q^{n} \\
h_{f}=K\left(Q_{0}^{n}+Q_{0}^{n-1} \Delta Q+\right. \\
\left.n(n-1) Q_{0}^{n-2} \Delta Q^{2}+\cdots\right)
\end{gathered}
$$

Y,

$$
\Delta Q=Q-Q_{0}
$$

Donde $h f$ representa la caída de presión, y $K$ denota una resistencia constante de la tubería. La caída de presión es usualmente relacionada al flujo en la tubería con la potencia característica, n. Los métodos de DarcyWeisbach y Hazen-Williams son comúnmente usados para definir los valores de $n$ y $K$ [17]. Asimismo, Q es el "flujo correcto", y $\Delta Q$ es el factor de corrección del flujo.

Si $\Delta Q$ es relativamente insignificante en comparación con $Q_{0}$, la ecuación 2 podría ser descrita como se enuncia en la ecuación 4.

$$
h_{f}=K\left(Q_{0}^{n}+Q_{0}^{n-1} \Delta Q\right)
$$

Para un grupo de sistemas tipo bucles, se tiene (véase ecuación 5).

$$
\begin{aligned}
\sum h_{f}=\sum K Q^{n} & =\sum K Q|Q|^{n-1} \\
& =\sum K Q_{0}\left|Q_{0}\right|^{n-1} \\
& +\Delta Q \sum k n k\left|Q_{0}\right|^{n-1} \\
& =0
\end{aligned}
$$

En la cual, $\Delta Q$ es asumido de igual manera para todas las secciones de tubería en un bucle. Las anotaciones de valor absoluto se han añadido para tener en cuenta la dirección de la sumatoria alrededor de un bucle dado. La ecuación 6 permite obtener $\Delta Q$.

$$
\Delta Q=-\frac{\sum K Q_{0}\left|Q_{0}\right|^{n-1}}{\sum n k\left|Q_{0}\right|^{n-1}}
$$

Posteriormente, $\Delta Q$ se aplica para actualizar el caudal en la ecuación 7 para cada sección de tubería en un bucle.

$$
Q_{0, \text { nuevo }} \sim Q=-Q_{0}+\Delta Q
$$

Para sistemas complejos de redes de tuberías, donde existen fuentes múltiples, y diferencias en la elevación, la ecuación 6 debe ser acondicionada para considerar dichos efectos (véase ecuación 8).

Y,

$$
\Delta Q=-\frac{\sum K Q_{0}\left|Q_{0}\right|^{n-1}+a}{\sum n k\left|Q_{0}\right|^{n-1}+b}
$$

$$
\begin{gathered}
a=\sum_{\text {perdidas }}\left(\frac{8 C_{e}}{g_{c} \pi^{2} D^{4}}\right) Q_{0}\left|Q_{0}\right| \\
b=\sum_{\text {perdidas }}\left(\frac{16 C_{e}}{g_{c} \pi^{2} D^{4}}\right)\left|Q_{0}\right|
\end{gathered}
$$

Donde $C_{e}$ es el coeficiente de pérdida menor, causado por un cambio en el área de sección transversal del flujo entre la tubería y la fuente. Para los sistemas de red de tuberías grandes, los efectos de entrada y salida pueden ser insignificantes cuando sus pérdidas de presión no son representativas en comparación con las caídas de presión de la tubería.

\subsection{Newton-Raphson}

El método de Newton-Raphson es un método numérico que permite resolver sistemas de ecuaciones matemáticas de manera simultánea. Su principal utilidad, según Pipes [18] radica en la facilidad que ofrece para resolver ecuaciones diferenciales cuando se conocen valores aproximados de los parámetros desconocidos deseados.

El objetivo de este método multivariable es linealizar los sistemas de ecuaciones mediante el uso de series de Taylor, de tal manera que el modelo resultante pueda ser resuelto a través de un determinado método de eliminación como el Gaussiano con sustitución hacia atrás.

Suponga que los caudales de un sistema de tuberías están representados mediante la formulación observada en la ecuación 11, el cual no es lineal y sus variables pueden ser aproximadas inicialmente como se muestra en la ecuación 12.

$$
\begin{gathered}
f_{1}\left(Q_{1}, Q_{2}, \ldots, Q_{n}\right)=0 \\
f_{2}\left(Q_{1}, Q_{2}, \ldots, Q_{n}\right)=0 \\
\vdots \\
f_{m}\left(Q_{1}, Q_{2}, \ldots, Q_{n}\right)=0 \\
Q_{1}=Q_{1}^{k}, Q_{2}=Q_{2}^{k}, \ldots, Q_{n}=Q_{n}^{k} .
\end{gathered}
$$


Entonces, al utilizar las series de Taylor se puede obtener una aproximación inicial de los valores, de la forma indicada en la ecuación 13.

$$
\begin{aligned}
& f_{i}\left(Q_{1}, Q_{2}, \ldots, Q_{n}\right)= \\
& \left.f_{i}\left(Q_{i}^{k}, Q_{2}^{k}, \ldots, Q_{n}^{k}\right) \sum_{j=1}^{n}\left(\frac{\partial f_{i}}{\partial Q_{i}}\right)\right|_{Q_{j}^{k}}\left(Q_{j} Q_{j}^{k}\right)=
\end{aligned}
$$

0

Este resultado puede ser representado matricialmente, como se muestra en la ecuación 14.

$$
\begin{aligned}
& \left.\left[\begin{array}{cccc}
\frac{\partial f_{1}^{k}}{\partial Q_{1}^{k}} & \frac{\partial f_{1}^{k}}{\partial Q_{2}^{k}} & \cdots & \frac{\partial f_{1}^{k}}{\partial Q_{n}^{k}} \\
\frac{\partial f_{2}^{k}}{\partial Q_{1}^{k}} & \frac{\partial f_{2}^{k}}{\partial Q_{2}^{k}} & \cdots & \frac{\partial f_{2}^{k}}{\partial Q_{n}^{k}} \\
\vdots & \vdots & & \vdots \\
\frac{\partial f_{n}^{k}}{\partial Q_{1}^{k}} & \frac{\partial f_{n}^{k}}{\partial Q_{2}^{k}} & \cdots & \frac{\partial f_{n}^{k}}{\partial Q_{n}^{k}}
\end{array}\right]_{m x n} \quad \begin{array}{c}
Q_{1}-Q_{1}^{k} \\
Q_{2}-Q_{2}^{k} \\
\vdots \\
Q_{n}-Q_{n}^{k}
\end{array}\right]_{n x 1} \\
& =-\left[\begin{array}{c}
f_{1}\left(Q_{i}^{k}, Q_{2}^{k}, \ldots, Q_{n}^{k}\right) \\
f_{2}\left(Q_{i}^{k}, Q_{2}^{k}, \ldots, Q_{n}^{k}\right) \\
\vdots \\
f_{m}\left(Q_{i}^{k}, Q_{2}^{k}, \ldots, Q_{n}^{k}\right)
\end{array}\right]_{m x 1}
\end{aligned}
$$

De tal manera que al reordenar se tiene lo expresado por la ecuación 15 .

$$
\begin{gathered}
{\left[\begin{array}{c}
Q_{1}-Q_{1}^{k} \\
Q_{2}-Q_{2}^{k} \\
\vdots \\
Q_{n}-Q_{n}^{k}
\end{array}\right]_{n x 1}=} \\
-\left[\begin{array}{cccc}
\frac{\partial f_{1}^{k}}{\partial Q_{1}^{k}} & \frac{\partial f_{1}^{k}}{\partial Q_{2}^{k}} & \ldots & \frac{\partial f_{1}^{k}}{\partial Q_{n}^{k}} \\
\frac{\partial f_{2}^{k}}{\partial Q_{1}^{k}} & \frac{\partial f_{2}^{k}}{\partial Q_{2}^{k}} & \ldots & \frac{\partial f_{2}^{k}}{\partial Q_{n}^{k}} \\
\vdots & \vdots & \ddots & \vdots \\
\frac{\partial f_{n}^{k}}{\partial Q_{1}^{k}} & \frac{\partial f_{n}^{k}}{\partial Q_{2}^{k}} & \ldots & \frac{\partial f_{n}^{k}}{\partial Q_{n}^{k}}
\end{array}\right]_{m x n} \\
{\left[\begin{array}{ccc}
f_{1}\left(Q_{i}^{k}, Q_{2}^{k}, \ldots, Q_{n}^{k}\right) \\
f_{2}\left(Q_{i}^{k}, Q_{2}^{k}, \ldots, Q_{n}^{k}\right) \\
\vdots \\
f_{m}\left(Q_{i}^{k}, Q_{2}^{k}, \ldots, Q_{n}^{k}\right)
\end{array}\right]_{m x 1}}
\end{gathered}
$$

En la ecuación 16 se pueden observar los valores corregidos para la iteración posterior.

$$
\left[\begin{array}{c}
Q_{1}^{k+1} \\
Q_{2}^{k+1} \\
\vdots \\
Q_{n}^{k+1}
\end{array}\right]_{n x 1}=\left[\begin{array}{c}
Q_{1}^{k} \\
Q_{2}^{k} \\
\vdots \\
Q_{n}^{k}
\end{array}\right]_{n x 1}+\left[\begin{array}{c}
Q_{1}-Q_{1}^{k} \\
Q_{2}-Q_{2}^{k} \\
\vdots \\
Q_{n}-Q_{n}^{k}
\end{array}\right]_{n x 1}
$$

De lo anterior se puede concluir que, a medida que los valores de la matriz $Q_{1}-Q_{1}^{k}$ tienden a cero, el método logrará alcanzar una convergencia, y el proceso iterativo habrá llegado a su fin.

\subsection{Leyes de Kirchhoff}

Como se mencionó anteriormente, estas leyes fueron propuestas inicialmente para el análisis de circuitos eléctricos, sin embargo, la experiencia ha demostrado que pueden ser aplicadas para el análisis de sistemas de tuberías.

La primera ley de Kirchhoff expresa que la suma algebraica de los flujos en cada nodo debe ser cero, es decir, los flujos de entrada y salida del nodo deben ser iguales, como se muestra en las ecuaciones 17 y 18 .

$$
\begin{gathered}
\sum Q=0 \\
\sum Q_{\text {entrada }}-\sum Q_{\text {salida }}=0
\end{gathered}
$$

Por otra parte, la segunda ley (expresada en la ecuación 19), enuncia que la suma algebraica de los diferenciales de presión en cada bucle cerrado debe ser cero.

$$
\sum \Delta P=0
$$

No obstante, es de notar que el diferencial de presión en cada nodo $\left(P_{j}-P_{k}\right)$ está relacionado al flujo a través de este, y dicha relación está determinada por la ecuación de balance de energía, como se observa en la ecuación 20 :

$$
\begin{aligned}
& P_{j}-P_{k}=\left(\left(Z_{k}-Z_{j}\right)+\left(\frac{8}{g_{c} \pi^{2} D^{4}}\right) Q^{2}+\right. \\
& \sum_{\text {tuberias }} K Q^{n}+ \\
& \sum_{\text {perdidas }}\left(\frac{8\left(C_{e}+C_{v}\right)}{g_{c} \pi^{2} D^{4}}\right) Q^{2}-\sum_{\text {bombas }}\left(A_{o}+\right. \\
& \left.\left.A_{1} Q+A_{2} Q^{2}\right)\right) \frac{\gamma g_{c}}{g}
\end{aligned}
$$

Donde $Z_{j}$ y $Z_{k}$ son las elevaciones de los puntos j y k; $\gamma$, la gravedad específica del fluido, y $\mathrm{g}$, la constante gravitacional.

\section{Formulación del modelo propuesto}

El modelo expuesto consta de dos componentes principales complementarios entre sí: 1) el modelo de flujo multifásico que indica el comportamiento del fluido en movimiento basado en la ecuación de balance de energía, y 2) el modelo de red de tuberías que predice los caudales y las presiones en cada punto del sistema, derivado de las leyes de Kirchhoff y la ecuación de balance de materia.

Con el fin de plantear el modelo se asumen las siguientes suposiciones: 
1. La temperatura promedio del fluido es constante a través del sistema.

2. Se presentan condiciones de estado estable.

3. Esquema de fluido "Black Oil".

4. Se ignoraron efectos de codos, uniones, etc.

\subsection{Modelo de flujo multifásico}

Para analizar el flujo multifásico, cualquier tipo de correlación puede ser usada en el modelo numérico propuesto; sin embargo, para lograr acoplarla es necesario realizar una reorganización previa de las ecuaciones formuladas, con el fin de expresar el caudal de fluidos en función de las presiones de entrada y salida.

El procedimiento a seguir es similar para cualquier correlación de flujo multifásico( $[19,20,21,22,23,5,24$, $6,7,25]$; no obstante, por cuestiones de extensión solo se mostrará el método de Beggs y Brill [7], correspondiente a las ecuaciones 21-36.

Partiendo de la ecuación 21 usada para calcular el gradiente de presión de líquido:

$$
\frac{\Delta P}{\Delta Z}=\frac{\left(\frac{g}{g_{c}} \sin \theta \rho_{m}+\frac{f_{t p} G_{m} v_{m}}{2 g_{c} d}\right)}{1-\frac{\left[\rho_{l} H_{l}+\rho_{g}\left(1-H_{l}\right)\right] v_{m} v_{s g}}{g_{c} P}}
$$

Se definen los caudales de líquido y gas en términos de ciertas constantes arbitrarias, como se muestran en las ecuaciones 22-25:

$$
\begin{gathered}
q_{g}=\left[3,27 * 10^{-7} Z\left(R-R_{s}\right) \frac{\bar{T}}{\bar{P}}\right] q \\
=C_{1} q \\
q_{o}=\left[6,49 * 10^{-5} B_{o}\right] q=C_{2} q \\
q_{w}=\left[6,49 * 10^{-5} B_{w} W O R\right] q=C_{3} q \\
q_{l}=q_{o}+q_{w}=\left(C_{2}+C_{3}\right) q=C_{4} q
\end{gathered}
$$

Adicionalmente, la densidad y el flujo másico de la mezcla, así como las velocidades reales y superficiales, también deben expresarse usando estas constantes, a partir de las relaciones evidenciadas en las ecuaciones 2629:

$$
\begin{gathered}
\rho_{m}=\rho_{l} H_{l}+\rho_{g}\left(1-H_{l}\right) \\
G_{m}=\left(\rho_{L} C_{4}+\rho_{g} C_{1}\right) q \\
v_{m}=\frac{C_{1}+C_{4}}{A_{p}} * q \\
v_{s g}=\frac{C_{1} q}{A_{p}} * q
\end{gathered}
$$

Al reemplazar las variables la ecuación 21 se convierte en:

$$
\frac{\left(\frac{g}{g_{c}} \sin \theta \rho_{m}+\frac{f_{t p}\left(\rho_{o} C_{4}+\rho_{g} C_{1}\right) q * \frac{C_{1}+C_{4}}{A_{p}} q}{2 g_{c} D}\right)}{1-\frac{\rho_{m} * \frac{\left(C_{1}+C_{4}\right) q}{A_{p}} * \frac{C_{1} q}{A_{p}}}{g_{c} P}}
$$

Posteriormente se reducen términos semejantes:

$$
\left.\frac{\left(\frac{\frac{\Delta P}{\Delta Z}=}{2 D g \sin \theta \rho_{m}+\frac{f_{t p}\left(\rho_{o} C_{4}+\rho_{g} C_{1}\right)\left(C_{1}+C_{4}\right) q^{2}}{A_{p}}}\right)}{\frac{g_{c} P-\frac{\rho_{m}\left(C_{1}+C_{4}\right) C_{1} q^{2}}{A_{p}^{2}}}{P}}\right)
$$

Operando las fracciones se obtiene:

$$
\begin{gathered}
\frac{\Delta P}{\Delta Z}= \\
\frac{2 D g \sin \theta \rho_{m} P A_{p}^{2}+f_{t p}\left(\rho_{o} C_{4}+\rho_{g} C_{1}\right)\left(C_{1}+C_{4}\right) q^{2} P A}{2 D g_{c} P A^{2}-2 D \rho_{m}\left(C_{1}+C_{4}\right) C_{1} q^{2}}
\end{gathered}
$$

Separando términos a lado y lado:

$$
\begin{aligned}
& \quad \Delta P\left(2 D g_{c} P A^{2}-2 D \rho_{m}\left(C_{1}+C_{4}\right) C_{1} q^{2}\right) \\
& =\Delta Z\left(2 D g \sin \theta \rho_{m} P A_{p}^{2}\right. \\
& \left.+f_{t p}\left(\rho_{o} C_{4}+\rho_{g} C_{1}\right)\left(C_{1}+C_{4}\right) q^{2} P A\right)
\end{aligned}
$$

Se agrupan términos semejantes y se factoriza $\mathrm{q}^{2}$ :

$$
\begin{aligned}
& q^{2}\left(-\Delta Z f_{t p}\left(\rho_{o} C_{4}+\rho_{g} C_{1}\right)\left(C_{1}+C_{4}\right) P A\right. \\
& \left.-2 D \rho_{m} \Delta P\left(C_{1}+C_{4}\right) C_{1}\right) \\
& =\left(2 \Delta Z D g \sin \theta \rho_{m} P A_{p}^{2}\right. \\
& \left.-2 \Delta P D g_{c} P A^{2}\right)
\end{aligned}
$$

Al despejar:

$$
=\frac{q^{2}}{-\Delta Z f_{t p}\left(\rho_{o} C_{4}+\rho_{g} C_{1}\right)\left(C_{1}+C_{4}\right) P A-2 D \rho_{m} \Delta P\left(C_{1}+C_{4}\right) C_{1}}
$$

Reorganizando y reduciendo términos: 


$$
=\frac{q^{2}}{\left(C_{1}+C_{4}\right)\left[\frac{L f_{t p}\left(\rho_{o} C_{4}+\rho_{g} C_{1}\right)}{2 D}+\frac{C_{1} \rho_{m}\left(P_{1}-P_{2}\right)}{P}\right.}
$$

De este punto en adelante la variable $\mathrm{q}^{2}$ será denotada como S.

A continuación, se evidencia una metodología para calcular el caudal a través de una tubería, considerando flujo bifásico. En esta, los primeros cuatro pasos son comunes para todas las correlaciones de flujo multifásico, sin embargo, a partir del quinto, el enfoque cambia de acuerdo con cada autor. En el presente estudio, se describirán las correlaciones de: 1) Beggs y Brill [7] (véanse ecuaciones 37-59), 2) Eaton et al. [5] (véanse ecuaciones 60-85), 2) Dukler [6] (véanse ecuaciones 86114).

1. Calcular la presión promedio a la entrada y la salida de la tubería (véase ecuación 37):

$$
\bar{P}=\frac{P_{1}+P_{2}}{2}
$$

2. Calcular todas las propiedades del fluido a la presión promedio: $\mathrm{Z}, \mathrm{Rs}, \mathrm{Bo}, \mathrm{Bg}$, densidades $\mathrm{y}$ viscosidades (se debe asumir una temperatura promedio en la tubería).

3. Calcular $\mathrm{q}_{\mathrm{g}}, \mathrm{q}_{\mathrm{o}}, \mathrm{q}_{\mathrm{w}}$ y $\mathrm{q}_{1}$ de acuerdo con las ecuaciones 22-25.

4. Obtener el hold-up de líquido no-slip, $\lambda$ (constante) mediante la ecuación 38.

$$
\lambda=\frac{q_{l}}{q_{g}+q_{l}}=\frac{C_{4}}{C_{1}+C_{4}}
$$

\subsubsection{Beggs y Brill [7]}

5. Calcular la viscosidad del líquido:

$$
\mu_{l}=\mu_{o}\left(\frac{1}{1+W O R}\right)+\mu_{w}\left(\frac{W O R}{1+W O R}\right)
$$

6. Calcular los siguientes parámetros:

$$
\begin{gathered}
\mu_{m}=\lambda * \mu_{l}+(1-\lambda) * \mu_{g} \\
L_{1}=\exp (-4.62-3.757 * X-0.481 \\
\left.* X^{2}-0.0207 * X^{3}\right) \\
L_{2}=\exp (1.061-4.602 * X-1.609 \\
* X^{2}-0.179 * X^{3} \\
\left.+0.000635 * X^{5}\right)
\end{gathered}
$$

Donde: $X=\ln (\lambda)$

7. Calcular la densidad del líquido:

$$
\rho_{l}=\rho_{o}\left(\frac{1}{1+W O R}\right)+\rho_{w}\left(\frac{W O R}{1+W O R}\right)
$$

8. En la primera iteración asumir un valor de factor total de fricción $\left(\mathrm{f}_{\mathrm{sp}}=0,006\right)$ y calcular la densidad de la mezcla:

$$
\rho_{s p}^{(0)}=\lambda * \rho_{l}+(1-\lambda) * \rho_{g}
$$

9. Calcular el flujo total:

$$
q= \begin{cases}\sqrt{S} & \text { si } S \geq 0 \\ \sqrt{-S} & \text { si } S<0\end{cases}
$$

Donde:

$$
\begin{gathered}
S= \\
\frac{\left(144 *\left(P_{1}-P_{2}\right)-\rho_{s p} * L * \sin (\theta)\right) * 32.174 * A^{2}}{\left(C_{1}+C_{4} *\left[\frac{L * f_{S p}}{2 * D} *\left(C_{1} * \rho_{g}+C_{4} * \rho_{l}\right)+\frac{C_{1} * \rho_{s p}}{\bar{P}} *\left(P_{1}-P_{2}\right)\right]\right)}
\end{gathered}
$$

10. Calcular $\mathrm{N}_{\mathrm{FR}}, \mathrm{N}_{\mathrm{Re}}, \mathrm{N}_{\mathrm{LV}}$ :

$$
\begin{gathered}
N_{F R}=\frac{1}{32.174 * D} *\left[\frac{\left(C_{1}+C_{4}\right) * q}{A}\right]^{2} \\
N_{R e}=\frac{\left(\left(C_{1} * \rho_{g}+C_{4} * \rho_{l}\right) * q * D\right)}{A * \mu_{m}} \\
N_{L V}=1.938 * C_{4} * \frac{q}{A} *\left(\frac{\rho_{l}}{\sigma_{l}}\right)^{0.25}
\end{gathered}
$$

11. Determinar el patrón de flujo:

Segregado: $N_{F R}<L_{1}$ Intermitente: $L_{1}<N_{F R}<L_{2}$ Distribuido: $N_{F R}>L_{1}$ yy $N_{F R}>L_{2}$

12. Calcular el hold-up para tubería horizontal mostrado en la ecuación 50, usando las constantes de la tabla 1:

$$
H_{L}(0)=\frac{a * \lambda^{b}}{N_{F R}^{c}}
$$

Tabla 1. Constantes a, b y c para el cálculo de hold-up. Modificado de Beggs y Brill [7].

\begin{tabular}{cccc}
\hline $\begin{array}{c}\text { Régimen } \\
\text { de flujo }\end{array}$ & a & b & c \\
\hline Segregado & 0,98 & 0,4846 & 0,0868 \\
\hline Intermitente & 0,845 & 0,5351 & 0,0173 \\
\hline
\end{tabular}




\begin{tabular}{llll}
\hline Distribuido & 1,065 & 0,5824 & 0,0609
\end{tabular}

13. Calcular el factor de corrección de hold-up $\psi$ por inclinación, utilizando las constantes de la tabla 2:

$\Psi=1+C\left[\sin (1.8 * \theta)-\frac{1}{3} \sin ^{3}(1.8 *\right.$

$\theta)]$

Donde:

$$
C=(1-\lambda) * \ln \left(d * \lambda^{e} * N_{L V}^{f} * N_{F R}^{g}\right)
$$

Tabla 2. Constantes d, e, f y g para el cálculo de hold-up. Modificado de Beggs y Brill [7].

\begin{tabular}{|c|c|c|c|c|}
\hline $\begin{array}{l}\text { Régimen } \\
\text { de Flujo }\end{array}$ & d & $\mathbf{e}$ & $\mathbf{f}$ & g \\
\hline $\begin{array}{l}\text { Segregado } \\
\text { hacia arriba }\end{array}$ & 0.011 & -3.768 & 3.539 & -1.614 \\
\hline $\begin{array}{l}\text { Intermitente } \\
\text { hacia arriba }\end{array}$ & 2.96 & 0.305 & 0.4473 & 0.0978 \\
\hline $\begin{array}{l}\text { Distribuido } \\
\text { hacia arriba }\end{array}$ & \multicolumn{4}{|c|}{$\begin{array}{c}\text { No se corrige } \mathrm{C}=0, \Psi=1, H_{L} \neq \\
f(\theta)\end{array}$} \\
\hline $\begin{array}{c}\text { Todos los } \\
\text { patrones } \\
\text { hacia abajo }\end{array}$ & 4.70 & $\begin{array}{c}- \\
0.3692\end{array}$ & 0.1244 & 0.5056 \\
\hline
\end{tabular}

14. Calcular el hold-up de líquido y la densidad de la mezcla:

$$
\begin{gathered}
H_{L}(\theta)=H_{L}(0) * \Psi \\
\rho_{s p}=H_{L}(\theta) * \rho_{o} \\
+(1 \\
\left.-H_{L}(\theta)\right) \\
* \rho_{g}
\end{gathered}
$$

15. Calcular el factor de fricción sin deslizamiento:

$$
\begin{aligned}
& f_{n s} \\
& {\left[2 * \log \left(\frac{1}{4,5223 * \log N_{R e}-3,8215}\right)\right]^{2}}
\end{aligned}
$$

16. Calcular el factor total de fricción:

$$
f_{s p}=f_{n s} * e^{s}
$$

Donde:

$$
\left\{\begin{array}{c}
S= \\
\frac{\ln (2.2 * Y-1.2) \text { si } 1.0<Y<1.2}{\ln (Y)} \\
-0,0523+3,18 * \ln (Y)-0,8725 * \ln (Y))^{2}+0,01856 *(\ln (Y))^{4}
\end{array}\right.
$$

$$
Y=\frac{\lambda}{H_{L}^{2}(\theta)}
$$

17. Calcular la tasa de flujo a una nueva iteración utilizando las ecuaciones del paso 6.

18. Repetir hasta alcanzar la convergencia:

$$
\left|q^{(n+1)}-q^{(n)}\right|<\epsilon
$$

\subsubsection{Eaton et al. [5]}

1. Calcular $\rho_{L}$ (véase ecuación 43) y $\rho_{g}$ :

$$
\rho_{g}=\frac{0.074 * S G_{g} *(\bar{P}+14.7) * 520}{14.7 *(\bar{T}+460) * Z}
$$

2. Asumir un factor total de fricción $\mathrm{f}_{\mathrm{sp}}=0,006 \mathrm{y}$ calcular el flujo total:

$$
q= \begin{cases}\sqrt{S} & \text { si } S \geq 0 \\ \sqrt{-S} & \text { si } S<0\end{cases}
$$

Donde:

$$
\begin{aligned}
& S= \\
& \frac{48 * d *\left(P_{1}-P_{2}\right) * 32,174 * A_{p}^{2} *\left(1+\frac{D}{6 * L * f_{s p}}\right)}{L *\left(\rho_{L} C_{4}+\rho_{g} C_{1}\right) *\left(C_{4}+C_{1}\right) * f_{s p}}
\end{aligned}
$$

3. Calcular la viscosidad del líquido (véase ecuación 39).

4. Calcular $\mathrm{V}_{\mathrm{SL}}$ y $\mathrm{V}_{\mathrm{SG}}$ :

$$
\begin{aligned}
& V_{s L}=\frac{q_{L}}{A_{p}} \\
& V_{s g}=\frac{q_{g}}{A_{p}}
\end{aligned}
$$

5. Calcular $H_{\Psi}$ para $P_{1}$ y $P_{2}$ :

$$
\begin{aligned}
& H_{\Psi}=\frac{N_{L V}^{0.575}}{N_{g v^{*} N_{d}^{0.0277}}} *\left(\frac{P}{14.65}\right)^{0.05} * \\
& \left(\frac{N_{L}}{0.00226}\right)^{0.1}
\end{aligned}
$$

Donde:

$$
\begin{gathered}
N_{L v}=1.938 * V_{s L} *\left(\frac{\rho_{L}}{\sigma}\right)^{0.25} \\
N_{g v}=1.938 * V_{s g} *\left(\frac{\rho_{L}}{\sigma}\right)^{0.25} \\
N_{d}=\frac{(120.872 * d)}{12} *\left(\frac{\rho_{L}}{\sigma}\right)^{0.5}
\end{gathered}
$$




$$
N_{L}=0,15726 * \mu_{L} *\left(\frac{1}{\rho_{L} * \sigma^{3}}\right)^{0.25}
$$

6. Calcular $\mathrm{H}_{\psi}$, $\mathrm{V}_{\mathrm{L}}$ y $\mathrm{V}_{\mathrm{g}}$

$$
\begin{aligned}
\text { Si } 0.001< & H_{\Psi}<1.40 \text { entonces } \\
H_{L}=-1.708 * & H_{\Psi}^{4}+4.9826 * H_{\Psi}^{3} \\
& -5.4823 * H_{\Psi}^{2} \\
& +2.7618 * H_{\Psi} \\
& +0.0106
\end{aligned}
$$

Si $1.40 \leq H_{\Psi}<10$ entonces

$$
\begin{gathered}
H_{L}=-0.0034 * H_{\Psi}^{2}+0.0541 \\
* H_{\Psi} \\
+0.7523 \\
V_{L}=\frac{V_{s L}}{H_{L}} \\
V_{g}=\frac{V_{s g}}{H_{L}}
\end{gathered}
$$

7. Calcular $\mathrm{y}$ :

$$
\begin{aligned}
& y=(G R)^{0.5} *\left(\frac{d_{B}}{d}\right)^{1.25} * \\
& \frac{G_{T} * d}{\mu_{g} * 12 *\left(6.72 * 10^{-4}\right)}
\end{aligned}
$$

Donde:

$$
\begin{aligned}
& G R=\frac{W_{g}}{W_{m}} \\
& L R=\frac{W_{L}}{W_{m}} \\
& G_{T}=\frac{W_{m}}{A_{p} / 144} \\
& W_{L}=\rho_{L} * q_{L} \\
& W_{g}=\rho_{g} * q_{g} \\
& W_{m}=W_{L}+W_{g}
\end{aligned}
$$

Luego:

Si $1000 \leq y<100000$ entonces

$$
I=7 * 10^{6} * y^{\wedge}-1.664
$$

Si $100000 \leq y<600000$ entonces

$$
\begin{aligned}
& I=5 * 10^{-24} * y^{4}-8 * \\
& 10^{-18} * y^{3}+4 * 10^{-12} * y^{2}- \\
& 1 * 10^{-6} * y+0.0971
\end{aligned}
$$

Si $600000 \leq y<1728000$ entonces

$$
I=7.0525 * y^{0.487}
$$

Y finalmente:

$$
\begin{aligned}
f_{s p} & =\frac{I}{L R^{0.1}} \\
L R & =\frac{W_{L}}{W_{m}}
\end{aligned}
$$

8. Calcular la tasa de flujo q a una nueva iteración utilizando las ecuaciones del paso 2.

9. Repetir hasta alcanzar la convergencia (véase ecuación 59).

\subsubsection{Dukler [6]}

1. Calcular $\rho_{L} y \rho_{g}$ (véanse ecuaciones 43 y 60 , respectivamente)

2. Calcular la viscosidad del líquido (véase ecuación 39).

3. Calcular $\mathrm{v}_{\mathrm{m}}$ :

$$
V_{m}=\frac{q_{g}+q_{l}}{A_{p}}
$$

4. Calcular la viscosidad de la mezcla sin deslizamiento:

$$
\mu_{m}=\lambda * \mu_{l}+(1-\lambda) * \mu_{g}
$$

5. Calcular $\rho_{\mathrm{m}}$ :

$$
\rho_{m}=\rho_{l}\left(\frac{\lambda^{2}}{H_{L}}\right)+\rho_{g}\left(\frac{(1-\lambda)^{2}}{1-H_{L}}\right)
$$

6. Calcular NRe:

$$
N_{R e}=\frac{D * V_{m} * \rho_{m}}{12 *\left(6,72 * 10^{-4}\right) \mu_{m}}
$$

7. Calcular el hold-up de líquido:

- $\quad$ Para $0,1 \leq \lambda \leq 1,0$

$$
\begin{array}{rl}
H_{L}(0)=b_{0}+b_{1} & x+b_{2} x^{2} \\
& +b_{3} x^{3} \\
& +b_{4} x^{4}
\end{array}
$$

Donde:

$$
x=10 \lambda-2.107
$$

$$
\begin{gathered}
b_{0}= \\
0.469609-0.138040 Z-0.027481 Z^{2}
\end{gathered}
$$




$$
\begin{aligned}
& +0.003537 Z^{3}-0.024212 Z^{4} \\
& +0.01097 Z^{5} \\
& +0.027187 Z^{6}-0.019885 Z^{7} \\
& -0.004693 Z^{8} \\
& +0.004295 Z^{9} \\
& 0.106343 \\
& b_{1}= \\
& +0.001065 Z-0.00349 Z^{2} \\
& -0.002214 Z^{3}+0.002365 Z^{4} \\
& +0.000567 Z^{5}-0.000726 Z^{6} \\
& +0.000127 Z^{7} \\
& \begin{array}{c}
b_{2}= \\
-0.015214+0.004208 Z+ \\
0.006524 Z^{2} \\
+0.000246 Z^{3}-0.00127 Z^{4}- \\
0.00028 Z^{5}+0.000105 Z^{6} \\
b_{3}= \\
0.001994+0.000064 Z- \\
0.000572 Z^{2}-0.00002 Z^{3}
\end{array} \\
& b_{4}= \\
& -0.000144+0.000016 Z+ \\
& 0.000083 Z^{2}-0.000133 Z^{3} \\
& +0.000043 Z^{4}-0.000042 Z^{5} \\
& -0.000028 Z^{6}+0.000106 Z^{7} \\
& +0.000003 Z^{8}-0.000022 Z^{9}
\end{aligned}
$$

Donde,

$$
Z=\log (N R e)-4.0176
$$

- $\quad$ Para $0,01 \leq \lambda \leq 0,1$

$$
y L(o)=b 0+b 1 \lambda
$$

- $\quad$ Para $0.006 \leq \lambda \leq 0.01$

$$
\begin{aligned}
& b_{0}=0.7464444+ \\
& 0.402593 x-0.459559 x^{2}+ \\
& 0.112758 x^{3}-0.008571 x^{4} \\
& b_{1}=0.037791+ \\
& 0.091513 x+ \\
& 0.205683 x^{2}+ \\
& 0.390756 x^{3}+ \\
& 0.47075 x^{4}- \\
& 0.230195 x^{5}+0.023875 x^{6}
\end{aligned}
$$

- $\quad$ Para $0.003 \leq \lambda \leq 0.006$

$$
b_{0}
$$$$
=0.800301
$$$$
+0.386447 x-0.524572 x^{2}
$$$$
+0.140726 x^{3}-0.011543 x^{4}
$$

$$
\begin{aligned}
& b_{1}=0.110852+ \\
& 0.254436 x+ \\
& 0.54049 x^{2}+ \\
& 0.966715 x^{3}+ \\
& 1.080144 x^{4}- \\
& 0.594425 x^{5}+ \\
& 0.067371 x^{6}
\end{aligned}
$$

- $\quad$ Para $0.0017 \leq \lambda \leq 0.003$

$$
\begin{aligned}
& b_{0} \\
& =0.844298 \\
& +0.363485 x-0.575184 x^{2} \\
& +0.165097 x^{3}-0.014327 x^{4} \\
& b_{1}=5.37305+ \\
& 11.209496 x+ \\
& 13.672301 x^{2}-6.758896 x^{3}+ \\
& 0.71421 x^{4} \\
& \text { P Para } 0.001 \leq \lambda \leq 0.0017 \\
& b_{0}=0.6911545+ \\
& 0.260211 x-0.494243 x^{2}+ \\
& 0.155236 x^{3}-0.014659 x^{4}
\end{aligned}
$$

$$
\begin{aligned}
& b_{1} \\
& =107.430534 \\
& +62.558994 x-36.118309 x^{2} \\
& +1.293692 x^{3}+0.581947 x^{4}
\end{aligned}
$$

La variable independiente de los coeficientes $b_{0}$ $\mathrm{y} \mathrm{b}_{1}$ para el intervalo $0.001 \leq \lambda \leq 0.01$ es equivalente al logaritmo del $\mathrm{N}_{\mathrm{Re}}$, $\mathrm{y}$ esto es:

$$
x=\log \left(N_{R e}\right)
$$

8. Reemplazar el valor de hold-up en el punto $9 \mathrm{e}$ iterar hasta alcanzar convergencia. Es decir:

$$
\left|H_{L}^{(n+1)}-H_{L}^{(n)}\right|<\epsilon
$$

9. Asumir un factor total de fricción $\mathrm{f}_{\mathrm{sp}}=0,006 \mathrm{y}$ calcular el flujo total:

$$
q= \begin{cases}\sqrt{S} & \text { si } S \geq 0 \\ \sqrt{-S} & \text { si } S<0\end{cases}
$$

Donde: 


$$
S=\frac{12 * D *\left(P_{1}-P_{2}\right) * 32,174}{2 * L * \rho_{m} *\left(\frac{C_{4}+C_{1}}{A_{p}}\right) * f_{s p}}
$$

10. Obtener $f_{n}$ y $f_{t p} / f_{n}$ :

$$
\begin{aligned}
& f n=0.0056+0.5 N_{R e}^{-0.32} \\
& \frac{f_{s p}}{f_{n}} \\
& =1.076587-2.182034 x-0.937941 x^{2} \\
& -0.101785 x^{3}
\end{aligned}
$$

Donde:

$$
x=\log (\lambda)
$$

11. Calcular $\mathrm{f}_{\text {tp: }}$ :

$$
f_{s p}=\left(\frac{f_{s p}}{f_{n}}\right) f_{n}
$$

12. Calcular la tasa de flujo a una nueva iteración utilizando las ecuaciones del paso 13.

13. Repetir hasta alcanzar la convergencia (véase ecuación 59).

\subsection{Modelo de red de tuberías}

La construcción del modelo de red de tuberías depende en gran medida del establecimiento de una relación entre las presiones y los caudales que entran y salen de los nodos, entendiéndose estos como las conexiones entre los diferentes elementos que conforman el sistema. Dicha relación corresponde, en el caso de las bombas y compresores a las curvas de desempeño provistas por el fabricante, y en el caso de las tuberías a las ecuaciones de pérdida de energía, definidas en la sección anterior [2].

Con el fin de definir correctamente las ecuaciones de este, y de todos los sistemas en general además de garantizar su solución, ciertos parámetros deben ser tomados en cuenta:

1. Debe existir máximo una incógnita en cada nodo (i.e. ya sea la presión, ya sea el caudal).

2. Por lo menos una presión debe ser especificada en el sistema.

3. Flujos que salen de un nodo son asignados con signo negativo, mientras que aquellos que llegan a un nodo son asignados positivos.

4. El ángulo de cada tubería se considera positivo cuando el nodo con el número mayor se encuentra a una altura superior que el nodo con el número menor.

Una vez definidas las condiciones anteriores se realiza un balance de masa en cada uno de los nodos, utilizando la ecuación de continuidad, basada en la primera Ley de Kirchhoff (véase Ecuación 115):

$$
\sum_{j=1}^{l_{i}} q_{i j}+Q_{i}=0 \text { para } \mathrm{i}=1,2, \ldots, \mathrm{N}
$$

Siendo $l_{i}$ el número de tuberías conectado al nodo iésimo, $\mathrm{N}$ el número total de nodos en el sistema, $\mathrm{q}_{\mathrm{ij}}$ el flujo total en la tubería j-ésima conectada al nodo i-ésimo y $\mathrm{Q}_{\mathrm{i}}$ el caudal de entrada o salida al nodo.

Ahora bien, para poder calcular el flujo a través de una determinada tubería utilizando el modelo bifásico, se deben asumir las presiones en todos y cada uno de los nodos del sistema, a excepción de los nodos en donde la presión es conocida, ya que permanecerá constante durante el proceso. No obstante, si las suposiciones no son correctas, es muy probable que la Ecuación 115 no se cumpla, para lo cual Tian y Adewumi [3] brindan una solución a este problema aumentando o disminuyendo presión en el nodo, dependiendo de si el balance másico es positivo o negativo, respectivamente (véase ecuación 116).

$$
P_{i}^{k+1}=P_{1}^{k}+\beta_{i}\left[\sum_{j=1}^{l_{i}} q_{i j}+Q_{i}\right]
$$

La ecuación 116 constituye la base de cálculo de la metodología enunciada en este trabajo. Como se ha venido mencionando, el procedimiento para el análisis de redes de tuberías es un algoritmo de solución simultánea, que no involucra el uso de matrices, y en el cual se iteran las presiones hasta alcanzar una convergencia, interpretada mediante la ecuación 117:

$$
\left|\sum_{j=1}^{l_{i}} q_{i j}+Q_{i}\right|<\varepsilon
$$

El término $\beta$ i corresponde a un factor de aceleración que permite a la iteración confluir más rápidamente y está definido en la ecuación 118 :

$$
\beta_{i}=\operatorname{Min}\left|\frac{P_{i}-P_{j}}{q_{i j}}\right| \text { para } \mathrm{j}=1,2, \ldots, \mathrm{l}_{\mathrm{i}}
$$

Cabe resaltar que en caso de presentarse una situación donde una presión en un nodo es especificada y el caudal de entrada o salida necesita ser calculado, la ecuación 119 puede ser utilizada:

$$
\sum_{j=1}^{l_{i}} q_{i j}=-Q_{i}
$$

\section{Solución numérica}

El modelo enmarcado se compone básicamente de cuatro partes. Estas son: 1) la identificación de la red y el tratamiento de datos de entrada; 2) el modelo multifásico; 3) el modelo de red, y 4) el procedimiento iterativo simultáneo.

Para ilustrar la metodología de solución en la figura 1 se muestra un ejemplo sencillo de un sistema constituido por ocho tuberías y ocho nodos, basado en el trabajado Tian 
y Adewumi (1994) [3], el cual se analizará. Un resumen

de los resultados obtenidos se presenta en las tablas 3-6.

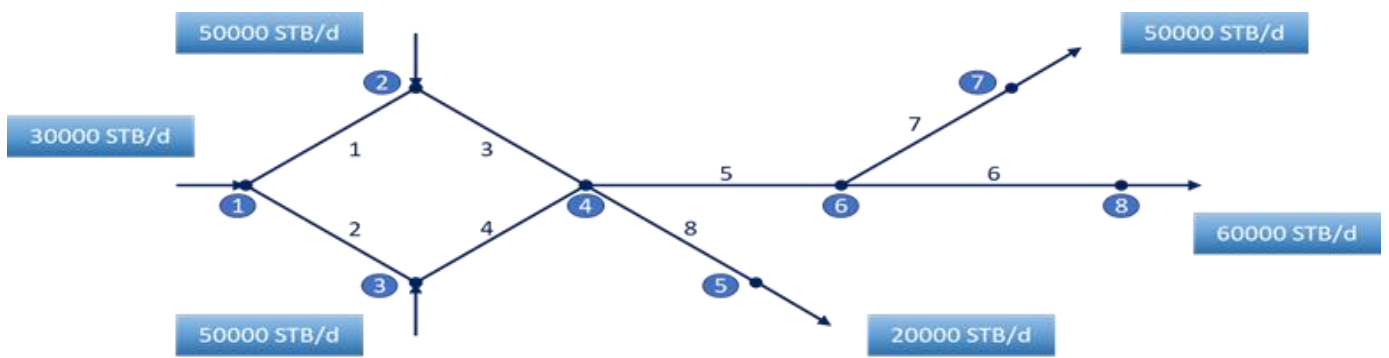

Figura 1. Requerimientos generales para modelar flujo multifásico. Modificado de Tian y Adewumi [2].

\subsection{Identificación de la red de tuberías}

Para iniciar el análisis del sistema la entrada de datos debe constar de tres componentes: 1) el número total de nodos y el número de tuberías conectadas a cada uno; 2) las propiedades del fluido y de las tuberías, y finalmente, 3) las presiones o flujos conocidos. A continuación, en la tabla 3 se encuentra un resumen de la relación existente entre los nodos y las tuberías del sistema.

Es importante aclarar que las propiedades de los fluidos transportados (i.e. aceite, agua y gas) deben ser calculadas mediante las correlaciones adecuadas; no obstante, valores constantes fueron asumidos para la fase líquida (i.e. agua y aceite) y la fase gaseosa, con el fin de agilizar el análisis, tal y como se muestra en la tabla 4.

En cuanto a las características de las líneas, fueron asumidas tuberías nuevas de acero. Adicionalmente, cabe destacar que los ángulos fueron referenciados como positivos cuando se movían de un lugar más bajo a un lugar más alto, siempre de un nodo inferior a un nodo superior; esto se puede ver ilustrado en la tabla 5.

Tabla 3. Relación entre nodos y tuberías del sistema.

\begin{tabular}{ccc}
\hline Nodo & Número de tuberías \\
\hline $\mathbf{2}$ & 2 & 2.1 \\
\hline & & 2.3 \\
\hline $\mathbf{3}$ & 2 & 3.2 \\
\hline & & 3.4 \\
\hline $\mathbf{4}$ & 4 & 4.3 \\
\hline & & 4.4 \\
\hline & & 4.5 \\
\hline & & 4.8 \\
\hline $\mathbf{5}$ & 1 & 5.8 \\
\hline $\mathbf{6}$ & 3 & 6.5 \\
\hline & & 6.6 \\
\hline & & 6.7 \\
\hline $\mathbf{7}$ & 1 & 7.7 \\
\hline $\mathbf{8}$ & 1 & 8.6 \\
\hline
\end{tabular}

Tabla 4. Propiedades del fluido a una presión promedio.

\begin{tabular}{cccc}
\hline$\gamma_{\mathbf{g}}$ & 0,65 & $\mathbf{T}$ & $50^{\circ} \mathrm{F}$ \\
\hline$\gamma_{\mathbf{l}}$ & 0,82 & WOR & $2 \mathrm{Bb} / \mathrm{STB}$ \\
\hline $\boldsymbol{\mu}_{\mathrm{g}}$ & $0,02 \mathrm{cp}$ & $\mathbf{G O R}$ & $1000 \mathrm{SCF} / \mathrm{STB}$ \\
\hline $\boldsymbol{\mu}_{\mathbf{0}}$ & $1,0 \mathrm{cp}$ & $\boldsymbol{\sigma}_{\mathbf{l}}$ & $30 \mathrm{dinas} / \mathrm{cm}$ \\
\hline $\boldsymbol{\mu}_{\mathbf{w}}$ & $1,0 \mathrm{cp}$ & $\mathbf{P}_{\mathbf{c}}$ & $660 \mathrm{psia}$ \\
\hline $\mathbf{B}_{\mathbf{o}}$ & 1,11 & $\mathbf{T}_{\mathbf{c}}$ & $440 \mathrm{R}$ \\
\hline $\mathbf{B}_{\mathbf{g}}$ & 0,0014 & $\mathbf{F . C}$. & 0,7236 \\
\hline $\mathbf{B}_{\mathbf{w}}$ & 1,01 & & \\
\hline
\end{tabular}

Tabla 5. Características de las tuberías del sistema.

\begin{tabular}{cccc}
\hline \multirow{2}{*}{$\begin{array}{c}\text { Número de } \\
\text { Tubería }\end{array}$} & \multicolumn{3}{c}{ Parámetros de Tubería } \\
\cline { 2 - 4 } & $\mathbf{L}_{\mathbf{i}}(\mathbf{f t})$ & $\mathbf{D}_{\mathbf{i}}(\mathbf{i n})$ & $\boldsymbol{\Theta}_{\mathbf{i}}$ \\
\hline $\mathbf{1}$ & 2000 & 6 & 1,5 \\
\hline $\mathbf{2}$ & 2000 & 6 & 1,5 \\
\hline $\mathbf{3}$ & 1500 & 9 & $-0,5$ \\
\hline $\mathbf{4}$ & 1500 & 9 & $-0,5$ \\
\hline $\mathbf{5}$ & 3000 & 12 & 0 \\
\hline $\mathbf{6}$ & 1000 & 9 & 2 \\
\hline $\mathbf{7}$ & 800 & 6 & 3,5 \\
\hline $\mathbf{8}$ & 1200 & 4 & -5 \\
\hline
\end{tabular}

Usando los datos anteriores, se puede llevar a cabo la identificación de la red, y seguidamente las ecuaciones características pueden ser generadas.

\subsection{Procedimiento iterativo}

Una vez terminada la fase de identificación se procede a calcular las presiones y caudales correspondientes a cada tubería de manera simultánea, usando los modelos de 
flujo tratados previamente, para posteriormente determinar las presiones y caudales en cada nodo mediante el modelo de red. La implementación del procedimiento iterativo es explicada detalladamente en el diagrama de flujo mostrado en la figura 2.

En la tabla 6 se muestra un resumen de los resultados conseguidos en la simulación. Se pueden apreciar en esta las presiones y caudales presentes en cada nodo.

\subsection{Discusión de resultados}

En este estudio, el modelo fue probado usando varios datos hipotéticos de una red de tuberías. Con el fin de verificar la validez de los resultados obtenido, estos fueron cotejados con los alcanzados por Tian y Adewumi [3]. La evaluación de los resultados es bastante simple, ya que las variables hidrodinámicas deben tener valores simétricos en la red propuesta. Los resultados de la predicción muestran que el modelo es capaz de predecir con gran precisión tanto la distribución de las presiones y flujos en cada nodo como el hold-up de líquido en cada tubería y el régimen de flujo, con la ventaja de tomar en cuenta una fase líquida compuesta no solo por un tipo de fluido (i.e. aceite) sino por dos fases inmiscibles (agua y aceite). Es de aclarar que el modelo propuesto es independiente a la calidad del crudo, tipo de fluido e inversiones de fases de mezclas agua-aceite, y de fenómenos tales como la corrosión [26], los cuales deben ser modelados por medios de correlaciones de fluidos para instancias Black oil, por lo tanto, la implementación del modelo planteado no está limitado por lo anteriores.

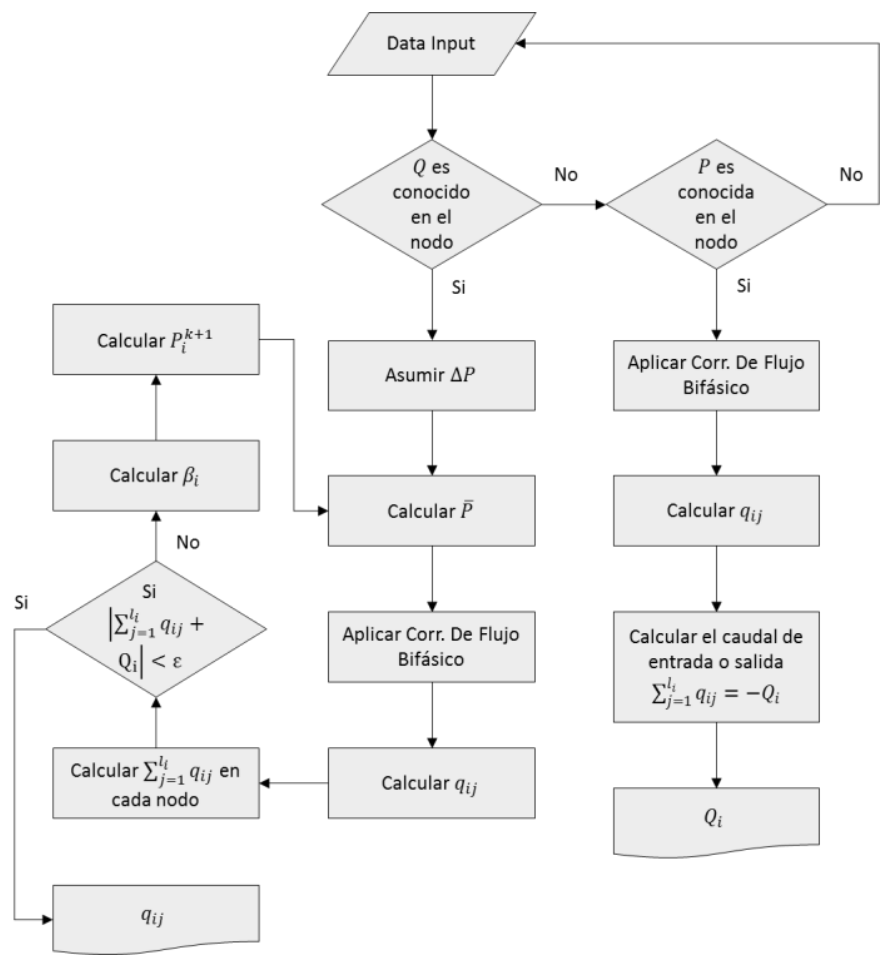

Figura 2. Diagrama de Flujo del modelo propuesto.

Tabla 6. Resultados obtenidos con el modelo propuesto. Modificado de Tian y Adewumi [2].

\begin{tabular}{ccccccc} 
Nodo & $\mathbf{Q}_{\mathbf{i}}[\mathbf{S T B} / \mathbf{d}]$ & $\begin{array}{c}\text { Número de } \\
\text { tuberías } \\
\text { conectadas }\end{array}$ & $\mathbf{P}_{\mathbf{i}}[\mathbf{p s i}]$ & Tubería & $\begin{array}{c}\mathbf{q i j} \\
{[\mathbf{S T B} / \mathbf{d}]}\end{array}$ & Patrón de flujo \\
\hline 1 & 30000 & 2 & $3000,00^{*}$ & 1 & 15000 & Distribuido \\
\hline 2 & 50000 & 2 & 2847,97 & 2 & 15000 & Distribuido \\
\hline 3 & 50000 & 2 & 2847,97 & 3 & 65000 & Distribuido \\
\hline 4 & 0 & 4 & 2652,33 & 4 & 65000 & Distribuido \\
\hline 5 & -20000 & 1 & 1707,88 & 5 & 110000 & Distribuido \\
\hline 6 & 0 & 3 & 2387,54 & 6 & 60000 & Distribuido \\
\hline 7 & -50000 & 1 & 1875,00 & 7 & 50000 & Distribuido \\
\hline 8 & 60000 & 1 & 2257,50 & 8 & 20000 & Distribuido \\
\hline
\end{tabular}




\subsection{Discusión de resultados}

En este estudio, el modelo fue probado usando varios datos hipotéticos de una red de tuberías. Con el fin de verificar la validez de los resultados obtenido, estos fueron cotejados con los alcanzados por Tian y Adewumi [3]. La evaluación de los resultados es bastante simple, ya que las variables hidrodinámicas deben tener valores simétricos en la red propuesta.

Los resultados de la predicción muestran que el modelo es capaz de predecir con gran precisión tanto la distribución de las presiones y flujos en cada nodo como el hold-up de líquido en cada tubería y el régimen de flujo, con la ventaja de tomar en cuenta una fase líquida compuesta no solo por un tipo de fluido (i.e. aceite) sino por dos fases inmiscibles (agua y aceite). Es de aclarar que el modelo propuesto es independiente a la calidad del crudo, tipo de fluido e inversiones de fases de mezclas agua-aceite, y de fenómenos tales como la corrosión [26], los cuales deben ser modelados por medios de correlaciones de fluidos para instancias Black oil, por lo tanto, la implementación del modelo planteado no está limitado por lo anteriores.

\section{Conclusiones}

En el presente estudio se desarrolló un nuevo modelo numérico, el cual permite el análisis y diseño de redes de tuberías con flujo multifásico, a través de la implementación de un algoritmo de resolución simultánea que no requiere del manejo algebraico de matrices.

Este modelo cuenta con diversas ventajas entre las que destacan su fácil utilización, la posibilidad de manejar presiones y caudales como parámetros de inicialización, además de que su aplicabilidad no se ve restringida por el tipo de configuración del sistema o los fluidos tratados, lo cual lo hace idóneo para la simulación de la mayoría de los sistemas actuales.

\section{Agradecimientos}

Los autores agradecen a la Universidad Industrial de Santander, al grupo de Modelamiento de Procesos de Hidrocarburos y a Petroleum Consulting Company S. A. S.

\section{Nomenclatura}

$\mathrm{A}=$ Área transversal de la tubería, $\mathrm{ft}^{2}$

$\mathrm{B}_{\mathrm{o}}=$ Factor volumétrico de formación del aceite, $\mathrm{Bb} / \mathrm{STB}$

$\mathrm{B}_{\mathrm{w}}=$ Factor volumétrico de formación del agua, Bb/STB

$C_{e}=$ Coeficiente de perdida menor.

$\mathrm{D}=$ Diámetro de la tubería, $\mathrm{ft}$

F.C. $=$ Factor de compresibilidad del gas, adim. $h_{f}=$ Caida de Presión.

$\mathrm{H}_{\mathrm{L}}(0)=$ Hold-up horizontal de líquido, adim.

$K=$ Constante referida a la resistencia en la tubería

$\mathrm{L}=$ Longitud de la tubería, $\mathrm{ft}$

$\mathrm{n}=$ Potencia característica de la tubería

$\mathrm{N}_{\mathrm{FR}}=$ Número de Froud, adim

$\mathrm{N}_{\mathrm{Re}}=$ Número de Reynolds, adim

$\mathrm{N}_{\mathrm{LV}}=$ Número de velocidad del líquido

$\mathrm{P}=$ Presión promedio, psia

$\mathrm{P}_{1}=$ Presión en el nodo inicial, psi

$\mathrm{P}_{2}=$ Presión en el nodo final, psi

$q$ = Caudal total a través de la tubería, STB/d

$\mathrm{q}_{\mathrm{g}}=$ caudal de gas, $\mathrm{ft}^{3} / \mathrm{s}$

$\mathrm{q}_{\mathrm{o}}=$ caudal de aceite, $\mathrm{ft}^{3} / \mathrm{s}$

$\mathrm{q}_{\mathrm{w}}=$ caudal de agua, $\mathrm{ft}^{3} / \mathrm{s}$

$\mathrm{q}_{\mathrm{l}}=$ caudal de líquido, $\mathrm{ft}^{3} / \mathrm{s}$

$\mathrm{Q} 0=$ Flujo inicial asumido.

$\mathrm{R}=$ Relación gas/aceite, $\mathrm{SCF} / \mathrm{STB}$

$\mathrm{R}_{\mathrm{s}}=$ Relación gas en solución/aceite, SCF/STB

$\mathrm{SG}_{\mathrm{g}}=$ Gravedad específica del gas, adim.

$\mathrm{T}=$ Temperatura promedio, $\mathrm{R}$

WOR = Relación agua/aceite, adim.

$\mathrm{Z}=$ Elevación.

$\rho_{\mathrm{o}}=$ densidad del aceite, $\mathrm{Lbm} / \mathrm{ft}^{3}$

$\rho_{\mathrm{w}}=$ densidad del agua, $\mathrm{Lbm} / \mathrm{ft}^{3}$

$\rho \mathrm{s}_{\mathrm{p}}=$ Densidad de la mezcla, $\mathrm{Lbm} / \mathrm{ft}^{3}$

$\rho_{\mathrm{l}}=$ Densidad del líquido, $\mathrm{Lbm} / \mathrm{ft}^{3}$

$\rho_{\mathrm{g}}=$ Densidad del gas, $\mathrm{Lbm} / \mathrm{ft}^{3}$

$\mu_{\mathrm{o}}=$ viscosidad de aceite, $\mathrm{cp}$

$\mu_{\mathrm{w}}=$ viscosidad de agua, $\mathrm{cp}$

$\mu_{\mathrm{m}}=$ Viscosidad de la mezcla, $\mathrm{cp}$

$\mu_{1}=$ Viscosidad del líquido, $\mathrm{cp}$

$\mu_{\mathrm{g}}=$ Viscosidad del gas, $\mathrm{cp}$

$\Theta=$ Ángulo de la tubería tomado desde el nodo menor al nodo mayor, grados

$\sigma_{l}=$ Tensión superficial del líquido

\section{Referencias}

[1] G. Valle, F. Romero y M. Cabarcas, Predicción de flujo multifásico en sistemas de recolección de crudo: descripción de requerimientos, Fuentes el Reventón Energético, v. 15, n. ${ }^{\circ}$ 1, pp. 87-99., 2017.

[2] L. Mucharam y M. Adewumi, A Compositional TwoPhase Flow Model for Analyzing and Designing Complex Pipeline Network Systems, Society of Petroleum Engineers, SPE 21562, 1990, p. 17.

[3] S. Tian y M. Adewumi, A New Algorithm for Analyzing and Designing Two-Phase Flow Pipeline Networks, Pennsylvania: Society of Petroleum Engineers, SPE 28177, 1994. 
[4] U. Shamir y C. Howard, «WATER DISTRIBUTION SYSTEMS ANALYSIS,» Journal of the Hydraulics Division , pp. 219-234, 1968.

[5] B. Eaton, D. Andrews, C. Knowles y K. Brwon, The Prediction of Flow Patterns, Liquid Holdup and Pressure Losses Occurring During Continuous Two-Phase Flow in Horizontal Pipelines., J. Pet. Technol, 19 (6), 815-828 (SPE Paper 1525)., 1967.

[6] A. Dukler, Gas Liquid Flow in Pipelines: I. Research Results, AGA-API Project NX-28, 1969.

[7] H. Beggs y J. Brill, A Study of Two-Phase Flow in Inclined Pipes, J. Pet. Technol., Trans., AIME, 25 (5), 607-617., 1973.

[8] T. Walski, Pipe Network Modeling, VIdeo Learning System For CYBERNET Software, Haestad Methods: Waterbury, 1994.

[9] J. Martinez y L. Puigjaner, A powerful improvement on the methodology for solving large-scale pipeline networks., Comput. Chem. Eng. 12 (2-3), 261-265., 1988.

[10] H. Cross, Analysis of Flow in Networks of Conduits or Conductors., Universityof Illinois Engineering Experiment Station, 1936.

[11] B. Gay y P. Middleton, The solution of pipe network problems, Chem. Eng. Sci, 26, 109-123., 1970.

[12] R. Sargent, The Decomposition of Systems of Procedures and Algebraic Equations in Numerical Analysis, Watson, G.A. (Ed.). Springer-Verlag, Berlin., 1978.

[13] M. Shacham, Decomposition of systems of nonlinear algebraic equations, AIChE J. 30 (1), 92-99., 1984.

[14] J. Krope, P. Trop y D. Goricanec, Flow pressure analysis of loop gas network, international journal of systems applications., Eng. Dev. 5 (4)., 2011.

[15] T. Altman y P. Boulos, Convergence of Newton method in nonlinear network analysis, Math. Comput. Model. 21 (4), 35-41., 1995.

[16] D. Brick, Iterative methods for looped network pipeline calculation. WaterResour, Manage. 25, 29152987., 2011.
[17] P. Bhave, Analysis of Flow in Water Distribution Networks, Technomic Publishing: Lancaster, PA, 1991.

[18] L. A. Pipes, Applied Mathematics for Engineers and Physicists, New York: McGraw-Hill, 1958.

[19] F. Poettmann y P. Carpenter, Multiphase Flow of Gas, Oil and Water Through Vertical Flow Strings., Drill. Prod. Pract., pp 257., 1952.

[20] O. Flanigan, Effect of Uphill Flow on Pressure Drop in Design of Two-Phase Gathering Systems., Oil Gas J., 1958.

[21] C. Hoogendorn, Gas-Liquid Flow in Horizontal Pipes, Chem. ENg. Sci., 9, 1959.

[22] H. Duns y N. Ros, Vertical Flow of Gas and Liquid Mixtures in Wells., In Proceedings of the Sixth World Petroleum Congress, Frankfurt, Germany; pp 451-465 (Section II, Paper 212.PD6)., 1963.

[23] A. Hagedorn y K. Brown, Experimental Study of Pressure Gradients Occurring During Continuous TwoPhase Flow in Small-Diameter Vertical Conduits., J. Pet. Technol, 17 (4), 475-484., 1965.

[24] J. Orkiszewski, Predicting Two-Phase Pressure Drops in Vertical Pipes., J. Pet. Technol., Trans. AIME, 19 (6), 829-838 (SPE Paper 1546-PA)., 1967.

[25] J. Mandhane, G. Gregory y K. Aziz, Critical Evaluation of Holdup Prediction Methods for Gas-Liquid Flow in Horinzontal Pipes, Society of Petroleum Engineers, SPE 5140, 1974.

[26] O. Gonzales-Estrada, J. Leal-Enciso y J. ReyesHerrera, "Análisis de integridad estructural de tuberías de material compuesto para el transporte de hidrocarburos por elementos finitos," Rev. UIS Ing., vol. 15, no. 2, pp. 105-116, 2016.2 Doi: https://doi.org/10.18273/revuin.v15n2-2016009 